\title{
Etiological trends in male central precocious puberty
}

\author{
Jisun Lee, MD, \\ Jinsup Kim, MD, \\ Aram Yang, MD, \\ Sung Yoon Cho, MD, PhD*, \\ Dong-Kyu Jin, MD, PhD*
}

Department of Pediatrics, Samsung Medical Center, Sungkyunkwan University School of Medicine, Seoul, Korea

Received: 5 September, 2017

Revised: 12 October, 2017

Accepted: 24 October, 2017

Address for correspondence:

Sung Yoon Cho, MD, PhD

Department of Pediatrics, Samsung

Medical Center, Sungkyunkwan

University School of Medicine,

81 Irwon-ro, Gangnam-gu, Seoul

06351, Korea

Tel: +82-2-6190-5227

Fax: +82-2-3410-0043

E-mail: nadri1217@naver.com

https://orcid.org/0000-0003-2913059X

Address for co-correspondence:

Dong-Kyu Jin, MD, PhD

Department of Pediatrics, Samsung Medical Center, Sungkyunkwan University School of Medicine, 81 Irwon-ro, Gangnam-gu, Seoul 06351, Korea

Tel: +82-2-3410-3525

Fax: +82-2-3410-0043

E-mail: jindk@skku.edu

https://orcid.org/0000-0003-41622706

*These authors contributed equally to this work.
Purpose: In the present study, the etiological trends in male central precocious puberty (CPP) were examined, and annual distribution was evaluated.

Methods: Seventy-one male CPP subjects who started puberty before 9 years of age were included in this study. All individuals were diagnosed as having CPP at Samsung Medical Center between 2001 and 2016. Chronological age at puberty onset, diagnosis of CPP, bone age, weight $(\mathrm{kg})$, height $(\mathrm{cm})$, puberty stage, brain magnetic resonance imaging findings, testosterone level, basal gonadotropin level, and gonadotropin level after gonadotropin releasing hormone stimulation were analyzed.

Results: The 71 patients were divided into 2 groups: idiopathic (group I) and organic (group II) when the lesion was identified as associated with the central nervous system (CNS) or when the patient received chemotherapy for non-CNS tumors before CPP diagnosis, respectively. Forty-four cases (62\%) were idiopathic, and 27 (38\%) were organic. The proportion of idiopathic CPP was higher than that of organic CPP during the study period. In $51.9 \%$ of organic cases, puberty started before 8 years of age, whereas it started after that age in $93.2 \%$ of the idiopathic cases.

Conclusion: In the present study, among all male CPP cases, $62 \%$ were idiopathic. The probability of idiopathic CPP prevalence was higher in males when the puberty onset was after 8 years of age with no history of cranial radiotherapy or chemotherapy.

Keywords: Precocious puberty, Male, Brain magnetic resonance imaging, Radiotherapy

\section{Introduction}

Precocious puberty (PP) is defined as the development of secondary sexual characteristics before the age of 8 years in girls and 9 years in boys. Depending on the presence of central activation of the hypothalamic-pituitary-gonadal axis, PP may be classified as gonadotropin dependent, also known as true or central PP (CPP), or gonadotropin independent, also known as pseudo-PP or peripheral PP. ${ }^{1)} \mathrm{CPP}$ may develop due to a previously identified or a new identifiable underlying central nervous system (CNS) disturbance (organic CPP) or by a nonidentifiable abnormality (idiopathic CPP). The causes of organic CPP include CNS irradiation, CNS tumors, CNS anomalies, postinfectious comorbidities, head trauma, and brain chronic inflammatory disorders. ${ }^{2,3)}$ The estimated incidence of PP is 1 in 5,000-100,000 individuals. The prevalence of PP is approximately 10 times higher in girls than in boys. ${ }^{4.5)}$

In contrast to girls, in whom most cases of CPP are idiopathic, an organic brain lesion is identified in the majority of boys diagnosed as having CPP. Compared with the prevalence of organic CPP in girls ( $8 \%-33 \%)$, that in boys is much higher $(40 \%-90 \%){ }^{6.77}$ Therefore, current guidelines recommend that brain magnetic resonance imaging (MRI) should be performed in boys diagnosed with CPP. According to studies conducted before the year 2000, the prevalence 
of neurogenic CPP in males has been revealed as $73 \%-94 \%,{ }^{8,9)}$ however, a wide range $(25 \%-83 \%)$ of this prevalence was observed in studies after the year $2000 .{ }^{9,10)}$ In a study conducted on 100 boys with CPP in Turkey in 2015, 26\% of them had organic causes while $74 \%$ of them had idiopathic CPP. ${ }^{10)}$ Another study conducted on 45 boys with CPP in Italy in 2000, $60 \%$ of them had idiopathic CPP while $40 \%$ of them organic causes. ${ }^{11)}$ Contrary to previous studies and endocrine references, in recent studies, idiopathic CPP in boys was more common than organic CPP. Moreover, recent studies have shown that the prevalence of idiopathic CPP has increased. ${ }^{12,13)}$

Despite the significance and rising prevalence of PP, there is still insufficient data on its etiology. Particularly, we need to identify whether the increase in CPP cases also applied to males since this has been reported in few previous studies.

This study aimed to examine the etiological trends in male CPP and evaluate the annual distribution of cases to identify with certainty that the number of idiopathic cases has increased recently.

\section{Materials and methods}

\section{Patients}

Herein, 71 male CPP subjects, started puberty before 9 years old, were included. All individuals were diagnosed as having CPP at the Samsung Medical Center from 2001 to 2016. The medical records were retrospectively reviewed. This study was reviewed and approved by the Institutional Review Board (IRB) of Samsung Medical Center (approval number: 2017-08-089001) and written informed consent was exempt from the IRB. Records on chronological age at puberty onset and diagnosis of CPP, bone age, weight $(\mathrm{kg})$, height $(\mathrm{cm})$, puberty stage, brain MRI findings, testosterone levels, basal gonadotropin levels, and gonadotropin levels after gonadotropin releasing hormone $(\mathrm{GnRH})$ stimulation were included. The patients' weight $(\mathrm{kg})$ and height $(\mathrm{cm})$ were measured with a digital body weighing scale and Harpenden stadiometer, respectively. Korean growth charts for children were implemented to interpret growth data, which were described as standard deviation scores (SDS). ${ }^{14}$ The body mass index (BMI) was represented as weight in kg/ height in meters squared. BMI-SDS were assessed according to the lambda-mu-sigma method using the 2007 Korean National Growth Charts. ${ }^{14)}$ Puberty stage was determined using the method of Marshall and Tanner. ${ }^{15)}$ Testicular volume was measured using a Prader orchidometer. Serum basal follicle stimulating hormone (FSH) and luteinizing hormone (LH) were measured by an immunoradiometric assay (DIAsource, Rue du Bosquet, Louvain-La-Neuve, Belgium). Serum testosterone levels were measured by radioimmunoassay (CIS BIO, Route Nationale, Saclay, France). The bone age was estimated using the Greulich and Pyle method. ${ }^{16)}$ Boys who had advanced bone age and testicular enlargement (volume $\geq 4 \mathrm{~mL}$ ) before 9 years of age from the medical record were diagnosed as CPP if the peak LH level was $\geq 5 \mathrm{IU} / \mathrm{L}$ in the GnRH stimulation test. GnRH test was performed as follows. We measured serum LH and FHS levels at $0,30,60,90$, and 120 minutes after intravenously administering $100 \mu \mathrm{g}$ of gonadorelin acetate (synthetically produced GnRH; Relefect, Sanofi-Aventis, Frankfurt am Main, Germany). Brain MRI was done in patients diagnosed with CPP. All 71 patients were divided into 2 groups: idiopathic (group I), organic (group II) when the lesion was identified as associated with CNS, or wherein the patients received chemotherapy for non-CNS tumors before CPP diagnosis.

\section{Statistical analysis}

Statistical analysis was done using SAS ver. 9.4 (SAS Institute Inc., Cary, NC). Data on continuous variables were presented as mean \pm standard deviation. Using the Student $t$-test, the importance of between-group differences was analyzed in terms of mean values when there were 2 independent groups. Fisher exact test was used to assess categorical variables. Moreover, minimum $P$-value approach was used to find the optimal cutoff age at which idiopathic CPP cases increased significantly when compared to organic cases.

We defined the possible cutoff age at puberty onset as 0.5 year unit within the range of 5-8.5 years, and divided the age into cutoffs or less and made them into binary variables at each cutoff value. The Fisher exact test was used to examine whether the associations between age (binary variable) and group variables (idiopathic group vs. organic groups) were significant. Then, we found the cutoff value of age at puberty onset with

Table 1. Clinical data and the laboratory results at diagnosis of central precocious puberty $(n=71)$

\begin{tabular}{lc}
\hline Variable & Value \\
\hline Chronological age at diagnosis (yr) & $8.5 \pm 1.7$ \\
Age at puberty onset (yr) & $7.7 \pm 1.5$ \\
Bone age (yr) & $10.2 \pm 2.0$ \\
BA-CA (yr) & $1.5 \pm 1.4$ \\
Weight-SDS & $0.2 \pm 1.4$ \\
Height-SDS & $0.8 \pm 1.3$ \\
BMI-SDS & $0.7 \pm 1.1$ \\
Basal LH (IU/L) & $1.6 \pm 1.3$ \\
Basal FHS (IU/L) & $2.8 \pm 2.5$ \\
Basal testosterone (ng/mL) & $1.2 \pm 1.9$ \\
Peak LH at GnRH stimulation (IU/L) & $14.7 \pm 8.6$ \\
Tanner stage, genital/pubic hair & \\
I & $0(0) / 0(0)$ \\
II & $57(80.3) / 53(74.7)$ \\
III & $14(19.7) / 16(22.5)$ \\
IV & $0(0) / 1(1.4)$ \\
V & $0(0) / 1(1.4)$ \\
\hline
\end{tabular}

Values are presented as mean \pm standard deviation or number (\%). BA, bone age; CA, chronological age; SDS, standard deviation score; BMI, body mass index; LH, luteinizing hormone; FSH, follicle-stimulating hormone; $\mathrm{GnRH}$, gonadotropin releasing hormone test. 
the smallest $P$-value. A $P$-value $<0.05$ was considered to indicate statistical significance.

\section{Results}

Clinical data and laboratory results were analyzed in 71 males with CPP (Table 1). The mean age at puberty onset was 7.2 \pm 1.5 years (age range, $2.8-8.9$ years). The mean bone age at diagnosis was $10.2 \pm 2.0$ years (age range, $3.5-13.5$ years). When puberty staging was done as per the method of Marshall and Tanner, most of them were at Tanner stage G2 (genitalia) at diagnosis. The pubic hair growth stage was P2-P5.

All patients who were diagnosed with CPP, performed brain MRI. Seventy-one patients were divided into 2 groups: idiopathic (group I), and organic group (group II). Fortyfour patients (62\%) were diagnosed as having idiopathic CPP. Twenty-seven patients (38\%) were diagnosed as having organic CPP. Among group II, only 4 cases (14.8\%) were diagnosed with non-CNS tumors and received chemotherapy before CPP diagnosis. These 4 patients received chemotherapy, wherein cyclophosphamide was commonly administered.

The causes of group II were listed (Table 2). Sixteen patients of group II had previously been treated for CNS diseases, whereas the other 7 patients revealed organic lesion on brain MRI after CPP diagnosis.

We compared the results of clinical data and laboratory results between groups I and II of male CPP (Table 3). The difference between the 2 groups with regard to the mean age at diagnosis, mean age of puberty onset and mean bone age at diagnosis was statistically significant.

The mean age at diagnosis was significantly younger in group II than in group I $(9.2 \pm 0.8$ years vs. $7.4 \pm 2.0$ years, $P<0.001)$. The mean age at puberty onset was significantly younger in group II than in group I $(8.3 \pm 0.7$ years vs. $6.7 \pm 2.0$ years, $P<0.001)$. The mean bone age at diagnosis was significantly advanced in group

Table 2. Etiology of central precocious puberty (CPP) in group II $(n=27)$

\begin{tabular}{l}
\hline Etiology of central precocious puberty \\
\hline Astrocytoma $(n=9)$ \\
Hamartoma ( $n=2)$ \\
Neuroblastoma with brain metastasis $(n=2)$ \\
Germinoma ( $n=1)$ \\
Retinoblastoma $(n=1)$ \\
D/Dx astrocytoma/hamartoma ( $n=1)$ \\
Pituitary macroadenoma $(n=1)$ \\
Arachnoid cyst $(n=1)$ \\
Partial empty sella ( $n=1)$ \\
Neuronal ceroid lipofuscinosis ( $n=1)$ \\
Epilepsy ( $n=3$ ) \\
Neuroblastoma ( $n=2)$ \\
Ewing's sarcoma $(n=1)$ \\
Burkitt lymphoma ( $n=1)$ \\
\hline Group II, organic CPP; D/Dx, differential diagnosis.
\end{tabular}

II than in group I $(10.5 \pm 1.2$ years vs. $8.8 \pm 2.6$ years, $P<0.003)$.

There were no significant differences between the 2 groups with respect to weight-SDS, BMI-SDS, basal LH, basal FSH, testosterone levels, and peak LH levels at the GnRH stimulation test.

In this study, we evaluated the annual distribution of male CPP cases to identify with certainty that the number of idiopathic cases has increased recently.

The cases were divided into 4 groups according to the following referral periods: 2001-2004, 2005-2008, 2009-2012, and 2013-2016. The mean age at puberty onset for each referral periods were $7.1 \pm 2.2,7.5 \pm 1.4,8.4 \pm 0.4$, and $7.6 \pm 1.7$, respectively. It showed that the number of male CPP cases increased over the years (Fig. 1). Idiopathic CPP was identified as the main proportion for this increase. Although the percentage of idiopathic CPP cases declined from $84.2 \%$ to $55.3 \%$ in the period 2013-2016 compared to the period 2009-2012, its proportion was higher than that of organic CPP during the entire study period. Fourteen of the 27 patients diagnosed as organic cases $(51.9 \%)$ were aged of puberty onset $<8.0$ years, and 41 of the 44 patients diagnosed as idiopathic cases (93.2\%) were aged of puberty onset $\geq 8.0$ years. Further, $82.4 \%$ of patients aged of puberty onset $<8.0$ years and diagnosed as having CPP had organic causes, whereas $75.9 \%$ of patients started puberty at an age of over 8.0 years and diagnosed as having CPP had idiopathic causes. We found the cutoff age at puberty onset using the minimum P-value approach. The cutoff age at puberty onset of 8.0 years was statistically significant $(P<0.001)$.

\section{Discussion}

According to recent studies, the prevalence of CPP

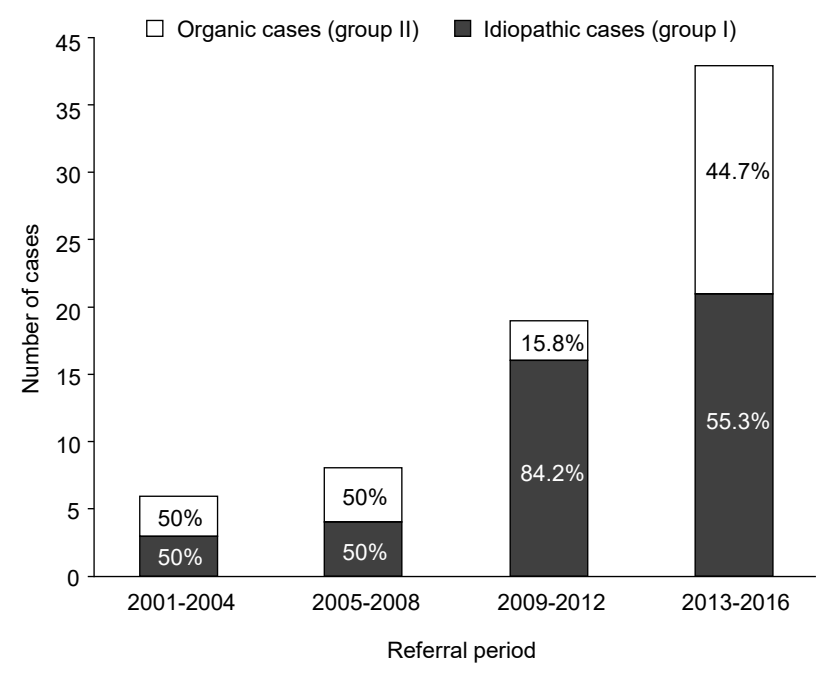

Fig. 1. The cases were divided into 4 groups according to the following referral periods: 2001-2004, 2005-2008, 2009-2012, and 2013-2016. It showed that the number of male central precocious puberty (CPP) cases increased over the years. Idiopathic CPP was identified as the main proportion for this increase. 
Table 3. Comparison of clinical data and laboratory results between groups I and II of male CPP who performed brain magnetic resonance imaging

\begin{tabular}{lccc}
\hline Variable & Group I (n=44) & Group II (n=27) & P-value \\
\hline Chronological age at diagnosis (yr) & $9.2 \pm 0.8$ & $7.4 \pm 2.0$ & $0.001^{\dagger}$ \\
Age at puberty onset (yr) & $8.3 \pm 0.7$ & $6.7 \pm 2.0$ & $0.001^{\dagger}$ \\
Bone age (yr) & $10.5 \pm 1.2$ & $8.8 \pm 2.6$ & $0.003^{\dagger}$ \\
BA-CA (yr) & $1.3 \pm 1.2$ & $1.7 \pm 1.8$ & $0.370^{\dagger}$ \\
Weight-SDS & $0.2 \pm 1.4$ & $0.1 \pm 1.4$ & $0.691^{\dagger}$ \\
Height-SDS & $0.7 \pm 1.3$ & $0.8 \pm 1.5$ & $0.819^{\dagger}$ \\
BMI-SDS & $0.8 \pm 1.0$ & $0.5 \pm 1.3$ & $0.373^{\dagger}$ \\
Basal_LH (IU/L) & $1.6 \pm 1.4$ & $0.733^{\dagger}$ \\
Basal_FHS (IU/L) & $2.6 \pm 2.3$ & $0.611^{\dagger}$ \\
Basal testosterone (ng/mL) & $1.0 \pm 1.9$ & $0.302^{\dagger}$ \\
Peak LH at GnRH stimulation (IU/L) & $13.3 \pm 7.4$ & $3.0 \pm 2.9$ & $0.079^{\dagger}$ \\
Tanner stage, genital/pubic hair & & $1.5 \pm 1.9$ & $1.000^{\ddagger} / 0.377^{\ddagger}$ \\
I & $0(0) / 0(0)$ & $17.0 \pm 10.0$ & \\
II & $35(79.5) / 34(77.3)$ & $22(81.5) / 19(70.4)$ & $5(18.5) / 6(22.2)$ \\
III & $9(20.5) / 10(22.7)$ & $0(0) / 1(3.7)$ & $0(0) / 1(3.7)$ \\
IV & $0(0) / 0(0)$ & $0(0) / 0(0)$ & \\
V & & & \\
\hline Values are presen &
\end{tabular}

Values are presented as mean \pm standard deviation or number (\%).

CPP, central precocious puberty; group I, idiopathic CPP, group II, organic CPP; BA, bone age; CA, chronological age; SDS, standard deviation score; BMI, body mass index; LH, luteinizing hormone; FSH, follicle-stimulating hormone; GnRH, gonadotropin releasing hormone test.

${ }^{\dagger}$ Student $t$-test. ${ }^{\ddagger}$ Fisher exact test.

is increasing and especially idiopathic CPP cases has increased. ${ }^{10,12,13,17)}$ This study examined whether the increase in CPP cases also applied to males. In our study on male CPP, the number of CPP in males shows an increasing trend, with idiopathic CPP identified as the main proportion for this increase.

Of the 71 male CPP patients in our study, idiopathic cases (group I), and organic cases (group II) were 62\% (44 of 71) and $38 \%$ ( 27 of 71 ), respectively.

In previous studies, hamartoma, optic nerve glioma, and astrocytoma have been more frequently associated with CPP development, whereas craniopharyngioma, Rathke's cysts, germinoma (unless there is secretion of beta-human chorionic gonadotropin $[\beta-h C G]$ in boys), and Langerhans cell histiocytosis were not. ${ }^{18)}$ Among them, hypothalamic hamartoma was the most commonly observed pathology in CNS, whereas herein, the most common CNS lesion was astrocytoma (9 of 27), with hamartoma (2 of 27) being the second most common. This may be the reason why the Samsung Medical Center is a tertiary university hospital and the proportion of cancer patients is relatively high.

Seven patients of group II had hamartoma $(\mathrm{n}=2)$, pituitary macroadenoma $(n=1)$, arachnoid cyst $(n=1)$, partial empty sella syndrome $(\mathrm{n}=1)$, germinoma $(\mathrm{n}=1)$, and suspicious hamartoma or astrocytoma on brain MRI without histopathologic confirmation $(\mathrm{n}=1)$. Among the 7 cases, 2 cases in which malignant brain tumors were found after CPP diagnosis were as follows. An 8.7-year-old boy presented with pubic hair growth was diagnosed as having CPP. He showed elevated $\beta$-hCG,
$55.7 \mathrm{mIU} / \mathrm{mL}$ (normal range, $<25 \mathrm{mIU} / \mathrm{mL}$ ), and brain MRI revealed a $2-\mathrm{cm}$ enhancing lesion involving left caudate nucleus. Histopathologic analysis confirmed a germinoma. A 2.8-yearold boy presented with uncontrolled seizure and significant pubic hair growth and was diagnosed as having CPP. A 1.5$\mathrm{cm}$ round mass in the hypothalamic area was identified by brain MRI. The brain lesion was suspected to be hamartoma or astrocytoma. However, histopathologic analysis was not done because he was not followed up after CPP diagnosis.

In group II, 6 patients were administered cranial radiotherapy before CPP diagnosis. The average radiation dose for each disease was 46 Gy in three astrocytoma patients, 25 Gy in one retinoblastoma patient, and $20 \mathrm{~Gy}$ in 2 neuroblastoma with brain metastasis patients.

The relationship between cranial radiotherapy and CPP has been confirmed in previous studies. ${ }^{19,20)}$ The mean age of onset of puberty in the 6 boys who underwent cranial radiotherapy for solid tumors was 7.5 years.

Herein, 4 patients who were diagnosed with non-CNS tumors and were administered chemotherapy before CPP diagnosis were classified into organic CPP cases (group II). The presentation of these 4 patients was not generally classified as the etiology of CPP in the endocrinology text book or other studies. ${ }^{2,621)}$ Previously, some studies have reported on the effect of administering only chemotherapy on timing and duration of puberty. ${ }^{22,23)}$ However, no reliable evidence for this effect exists. Moreover, few studies have reported on puberty timing in children treated for solid tumors outside the CNS. ${ }^{22)}$ Four patients in group II received chemotherapy which was used 
with $106 \mathrm{~B}$ regimen in one lymphoma patient, ICE/CCG321P2 regimen in 2 neuroblastoma patients, CCG321P2 regimen in one Ewing's sarcoma patient. Cyclophosphamide was commonly administered in 4 patients as the chemotherapy regimen. Particularly, alkylating agents can induce germ cell depletion in both sexes. This may cause delay or absence of puberty in girls, while most boys will have normal puberty despite the increased $\mathrm{LH}^{22)}$ Further studies are needed to determine if chemotherapy such as cyclophosphamide could be associated with male CPP.

Our study demonstrated that idiopathic CPP (62\%) was more common than organic CPP cases (38 \%) in boys. Compared to idiopathic CPP cases, organic CPP cases indicated a lower age at diagnosis, earlier onset of puberty and more advanced bone age. Our findings are supported by studies of Alikasifoglu et al. ${ }^{10)}$ and De Sanctis et al. ${ }^{11)}$ studies, both reporting younger age at diagnosis, earlier onset of puberty and greater bone age advancement in the neurogenic CPP group than in the idiopathic CPP group.

It is unclear whether the increase in idiopathic CPP prevalence is the result of an actual increase in the number of CPP patients. A possible explanation as to why idiopathic $\mathrm{CPP}$ is more common in recently is that its increase may be associated with improved nutritional supplementation and the effects of endocrine-disrupting chemicals. ${ }^{24)}$ Although consistent evidence supports that higher adiposity is associated with earlier pubertal maturation in girls, ${ }^{25)}$ a similar relationship in boys remains controversial. ${ }^{26,27)}$ However, recent studies reported that the effect of obesity on early puberty in boys. In a study conducted on 2,065 boys in Sweden, higher BMI scores related earlier puberty. ${ }^{28)}$ In a study conducted on 1,528 boys in Denmark, increasing BMI $z$-scores lead to an earlier onset of puberty. ${ }^{29)}$ In our study, idiopathic CPP cases had higher weightSDS and BMI-SDS than organic cases, although no significant difference was found.

Besides, in the last 10 years, reasons for increased reports of this condition can be attributed to better timely identification of the attributes due to improved recognition of CPP among general practitioners and an increase in parent's awareness regarding early puberty via the information at their disposal through media and internet.

There are some limitations of our study. The data on male CPP from one tertiary hospital could be deviated. We found that the proportion of organic in the male CPP cases was 38\%. However, this ratio is derived from the patients for whom brain MRI was performed, which is a limitation in the accurate evaluation of etiology of male CPP.

In conclusion, the number of male CPP shows an increasing trend over the past 15 years. The increase of idiopathic CPP cases was the main proportion of the overall increase in male CPP. The results from this study suggest that possible association factors for the presence of organic causes of CPP are young age at diagnosis, earlier onset of puberty, advanced bone age at CPP diagnosis, and the presence of other associated CNS disorders. When CPP is diagnosed in a boy before the age at puberty onset of 8.0, the cutoff age for higher probability of organic causes, we recommend performing brain MRI. In addition, when CPP is diagnosed in a boy between the age at puberty onset of 8-9, after the cutoff age for higher probability of organic causes, brain MRI should be considered. However, further studies are needed to evaluate the factors affecting early puberty, including genetic traits, nutrition, and environmental and psychosocial factors, that could explain increasing prevalence of CPP in our male patients.

\section{Conflict of interest}

No potential conflict of interest relevant to this article was reported.

\section{Acknowledgments}

This study was supported by a grant from Samsung Medical Center (\#GFO217006).

\section{References}

1. Berberoğlu M. Precocious puberty and normal variant puberty: definition, etiology, diagnosis and current management. J Clin Res Pediatr Endocrinol 2009;1:164-74.

2. Stephen MD, Zage PE, Waguespack SG. Gonadotropindependent precocious puberty: neoplastic causes and endocrine considerations. Int J Pediatr Endocrinol 2011;2011:184502.

3. Rohani F, Salehpur S, Saffari F. Etiology of precocious puberty, 10 years study in Endocrine Reserch Centre (Firouzgar), Tehran. Iran J Reprod Med 2012;10:1-6.

4. Kalantaridou SN, Chrousos GP. Clinical review 148: monogenic disorders of puberty. J Clin Endocrinol Metab 2002;87:2481-94.

5. Lee PA KJ. Precocious puberty. In: Pescovitz OH, Eugster EA, editors. Textbook of pediatric endocrinology. Philadelphia (PA): Lippincott Williams \&Wilkins, 2004:316-30.

6. Carel JC, Léger J. Clinical practice. Precocious puberty. N Engl J Med 2008;358:2366-77.

7. Carel JC, Lahlou N, Roger M, Chaussain JL. Precocious puberty and statural growth. Hum Reprod Update 2004;10:135-47.

8. Pescovitz OH, Comite F, Hench K, Barnes K, McNemar A, Foster $\mathrm{C}$, et al. The NIH experience with precocious puberty: diagnostic subgroups and response to short-term luteinizing hormone releasing hormone analogue therapy. J Pediatr 1986;108:47-54.

9. Pigneur B, Trivin C, Brauner R. Idiopathic central precocious puberty in 28 boys. Med Sci Monit 2008;14:CR10-14.

10. Alikasifoglu A, Vuralli D, Gonc EN, Ozon A, Kandemir N. Changing etiological trends in male precocious puberty: 
evaluation of 100 cases with central precocious puberty over the last decade. Horm Res Paediatr 2015;83:340-4.

11. De Sanctis V, Corrias A, Rizzo V, Bertelloni S, Urso L, Galluzzi F, et al. Etiology of central precocious puberty in males: the results of the Italian Study Group for Physiopathology of Puberty. J Pediatr Endocrinol Metab 2000;13 Suppl 1:687-93.

12. Bajpai A, Menon PS. Contemporary issues in precocious puberty. Indian J Endocrinol Metab 2011;15 Suppl 3:S1729.

13. Cisternino M, Arrigo T, Pasquino AM, Tinelli C, Antoniazzi F, Beduschi L, et al. Etiology and age incidence of precocious puberty in girls: a multicentric study. J Pediatr Endocrinol Metab 2000;13:695-701.

14. Moon JS, Lee SY, Nam CM, Choi JM, Choe BK, Seo JW, et al. 2007 Korean National Growth Charts: review of developmental process and an outlook. Korean J Pediatr 2008;51:1-25.

15. Marshall WA, Tanner JM. Variations in the pattern of pubertal changes in boys. Arch Dis Child 1970;45:13-23.

16. Milner GR, Levick RK, Kay R. Assessment of bone age: a comparison of the Greulich and Pyle, and the Tanner and Whitehouse methods. Clin Radiol 1986;37:119-21.

17. Kim SH, Huh K, Won S, Lee KW, Park MJ. A Significant increase in the incidence of central precocious puberty among Korean girls from 2004 to 2010. PLoS One 2015; 10:e0141844.

18. Stanhope R. Central precocious puberty and occult intracranial tumours. Clin Endocrinol (Oxf) 2001;54:2878 .

19. Leiper AD, Stanhope R, Kitching P, Chessells JM. Precocious and premature puberty associated with treatment of acute lymphoblastic leukaemia. Arch Dis Child 1987;62:1107-12.
20. Oberfield SE, Soranno D, Nirenberg A, Heller G, Allen JC, David R, et al. Age at onset of puberty following high-dose central nervous system radiation therapy. Arch Pediatr Adolesc Med 1996;150:589-92.

21. Spoudeas HA. Growth and endocrine function after chemotherapy and radiotherapy in childhood. Eur J Cancer 2002;38:1748-59.

22. Müller J. Disturbance of pubertal development after cancer treatment. Best Pract Res Clin Endocrinol Metab 200r;16:91-103.

23. Schwartz CL. Long-term survivors of childhood cancer: the late effects of therapy. Oncologist 1999;4:45-54.

24. Parent AS, Rasier G, Gerard A, Heger S, Roth C, Mastronardi C, et al. Early onset of puberty: tracking genetic and environmental factors. Horm Res 2005;64 Suppl 2:41-7.

25. Sørensen K, Mouritsen A, Aksglaede L, Hagen CP, Mogensen SS, Juul A. Recent secular trends in pubertal timing: implications for evaluation and diagnosis of precocious puberty. Horm Res Paediatr 2012;77:137-45.

26. Laron Z. Is obesity associated with early sexual maturation? Pediatrics 2004;113(1 Pt 1):171-2.

27. Ribeiro J, Santos P, Duarte J, Mota J. Association between overweight and early sexual maturation in Portuguese boys and girls. Ann Hum Biol 2006;33:55-63.

28. He Q, Karlberg J. Bmi in childhood and its association with height gain, timing of puberty, and final height. Pediatr Res 2001;49:244-51.

29. Sørensen K, Aksglaede L, Petersen JH, Juul A. Recent changes in pubertal timing in healthy Danish boys associations with body mass index. J Clin Endocrinol Metab 2010;95:263-70. 